\title{
The Effects of Wearing Lower-Body Compression Garments During Endurance Cycling
}

\section{Aaron T. Scanlan, Benjamin J. Dascombe, Peter R.J. Reaburn, and Mark Osborne}

\begin{abstract}
Purpose: The present investigation examined the physiological and performance effects of lower-body compression garments (LBCG) during a one-hour cycling timetrial in well-trained cyclists. Methods: Twelve well-trained male cyclists ([mean \pm SD] age: $20.5 \pm 3.6$ years; height: $177.5 \pm 4.9 \mathrm{~cm}$; body mass: $70.5 \pm 7.5 \mathrm{~kg} ; \mathrm{V}_{\mathrm{O} 2 \max }$ : $55.2 \pm 6.8 \mathrm{~mL} \cdot \mathrm{kg}^{-1} \cdot \mathrm{min}^{-1}$ ) volunteered for the study. Each subject completed two randomly ordered stepwise incremental tests and two randomly ordered one-hour time trials (1HTT) wearing either full-length SportSkins Classic LBCG or underwear briefs (control). Blood lactate concentration ([BLa $\left.\left.{ }^{-}\right]\right)$, heart rate (HR), oxygen consumption $\left(\mathrm{V}_{\mathrm{O} 2}\right)$ and muscle oxygenation (mOxy) were recorded throughout each test. Indicators of cycling endurance performance were anaerobic threshold (AnT) and $\mathrm{V}_{\mathrm{O} 2 \max }$ values from the incremental test, and mean power (W), peak power (W), and total work $(\mathrm{kJ})$ from the 1HTT. Magnitude-based inferences were used to determine if LBCG demonstrated any performance and/or physiological benefits. Results: A likely practically significant increase $\left(86 \%: 12 \%: 2 \% ; \eta^{2}=0.6\right)$ in power output at AnT was observed in the LBCG condition (CONT: $245.9 \pm 55.7 \mathrm{~W}$; LBCG: $259.8 \pm$ $44.6 \mathrm{~W})$. Further, a possible practically significant improvement $\left(78 \%: 19 \%: 3 \% ; \eta^{2}=\right.$ $0.6)$ was reported in muscle oxygenation economy $\left(\mathrm{W} \cdot \% \mathrm{mOxy}^{-1}\right)$ across the $1 \mathrm{HTT}$ (mOxy: CONT: $52.2 \pm 12.2 \%$; LBCG: $57.3 \pm 8.2 \%$ ). Conclusions: The present results demonstrated limited physiological benefits and no performance enhancement through wearing LBCG during a cycling time trial.
\end{abstract}

Keywords: compression garments, physiology, performance, near-infrared spectroscopy, bicycling

Historically, graduated compression garments (CG) have been used in treating various circulatory conditions in clinical patients. ${ }^{1,2}$ More recently, the use of CG has been adopted by athletes for potential performance and recovery benefits. , $^{3,4}$ Recent research has also suggested that wearing CG improves heat tolerance ${ }^{4,5}$

Scanlan and Reaburn are with the Department of Health and Human Performance, Central Queensland University, Rockhampton, Queensland, Australia; Dascombe is with the Western Australian Institute of Sport, Perth, Western Australia, Australia; and Osbourne is with the Queensland Academy of Sport, Brisbane, Queensland, Australia 
and may assist in injury prevention ${ }^{5,6}$ for athletes. The proposed mechanisms associated with wearing $\mathrm{CG}$ responsible for these benefits appear to be related to circulatory enhancements. ${ }^{3}$

Graduated CG provide varied levels of pressure across specific body segments with the compressive forces typically decreasing from the distal to proximal portions of isolated limbs. The pressure gradient applied by $\mathrm{CG}$ has been suggested to improve both venous blood flow and venous return. ${ }^{7,8}$ The increased venous flow may increase preloading of the heart during diastole, and result in stronger ventricular contractions through increased stretching of cardiac muscle tissue. This may lead to consequent increases in stroke volume and cardiac output via the Frank-Starling mechanism. ${ }^{9}$ Researchers have proposed that this sequence of circulatory events may result in improved endurance performance with wearing $\mathrm{CG}^{3}$; however, this relationship remains largely unsubstantiated.

Previously, the limited available CG research has largely focused on physiological and performance responses during endurance running. ${ }^{10-12}$ These studies have shown $\mathrm{CG}$ to offer beneficial metabolic responses, ${ }^{11}$ but no performance improvements. ${ }^{10,12}$ However, lower-body CG (LBCG) were recently observed to significantly enhance running economy during submaximal exercise in six trained runners. ${ }^{11}$ It was reported that participants wearing LBCG had a significantly $(P<$ .05) lower aerobic energy cost compared with loose-fitting shorts when running at $12 \mathrm{~km} \cdot \mathrm{h}^{-1}$ for a three-minute period. The researchers hypothesized that improvements in circulation and reductions in muscle oscillation may have promoted the observed economy improvements; however, these claims were not supported by empirical data.

When examining endurance running performance, LBCG have been observed to offer no significant benefits. Recent research demonstrated knee-length LBCG to exert no significant changes in $10-\mathrm{km}$ running time in fourteen well-trained endurance athletes. ${ }^{10}$ Furthermore, earlier work reported full-length LBCG to produce a significant $(P<.05)$ 31-s detriment in $5-\mathrm{km}$ run time in ten high-performance male endurance runners. ${ }^{12}$ It was suggested that the decrease in running performance was a result of reductions in stride length due to a restricted range of motion from the compressive forces of the LBCG.

Despite these observations, the use of CG among endurance athletes during training and performance remains popular. Furthermore, the effects of CG during non-running-based endurance activity, such as cycling, remain uninvestigated. Given the previously reported detrimental performance responses with CG during endurance running, it is hypothesized that wearing CG during endurance cycling will not elicit any beneficial changes in performance. Therefore, the purpose of the current study was to investigate the effects of wearing LBCG on physiological and performance responses during endurance cycling.

\section{Methods}

\section{Participants}

Twelve well-trained male cyclists ([mean $\pm S D$ ] age: $20.5 \pm 3.6$ years; height: $177.5 \pm 4.9 \mathrm{~cm}$; body mass: $70.5 \pm 7.5 \mathrm{~kg}$; maximal aerobic power $\left(\mathrm{V}_{\mathrm{O} 2 \max }\right): 55.2$ 
$\pm 6.8 \mathrm{~mL} \cdot \mathrm{kg}^{-1} \cdot \mathrm{min}^{-1}$ ) participated in the current study. All participants were competitive cyclists performing at least $300 \mathrm{~km}$ of cycling per week. Before testing, each subject was informed of the procedures and provided their written consent. Participants were also screened for any medical contraindications that may have excluded them from participation. All research practices were granted approval by the Central Queensland University Human Ethics Committee.

\section{Compression Garments}

The LBCG used were unisex full-length CG (SportSkins Classic, Skins, Campbelltown, NSW) comprised of 76\% Nylon and Meryl Microfiber and 24\% Roica Spandex. Each garment was custom-fit using a mathematical algorithm provided by the manufacturer and based on subject stature and body mass. The LBCG covered the lower limb from the superior aspect of the medial malleolus of the ankle to the superior aspect of the iliac crest. The LBCG were hypercompressive with the fabric expanding to almost $100 \%$ of body dimensions while exerting graded compressive forces on the underlying musculature. Compression over the musculature was measured using a Kikuhime pressure monitor (TT MediTrade, Sorø, Denmark). The LBCG exerted average pressure levels of $9.1 \pm 2.2,14.9 \pm 2.3$, $17.3 \pm 3.0$, and $19.5 \pm 3.4 \mathrm{mmHg}$ over the main body of the posterior gluteus maximus, vastus lateralis, medial gastrocnemius, and medial ankle, respectively (Figure 1). The control condition involved the use of regular, loose underwear briefs (Brief Cotton, Jockey International, Australia), with minimal compression applied over the leg musculature.

\section{Exercise Testing}

Throughout the testing procedures, all participants were instructed to maintain their regular training regimen and eat a high-carbohydrate diet. Participants were required to maintain training and diet diaries to ensure that these factors were standardized across the testing duration. All exercise testing sessions were separated by at least $48 \mathrm{~h}$ and were performed at the same time of day to avoid circadian variances. Participants were asked not to perform high-intensity training in the 24-h period before testing. All exercise testing was completed on an electromagnetically braked cycle ergometer (Excalibur, Lode, Groningen, The Netherlands). During test familiarization, each cyclist's position was matched to their own personal bicycle configuration. Participants wore their own cycling shoes and cleats throughout all exercise testing. Participants were required to maintain a cadence of between 90 to 100 RPM. All tests were completed within standardized laboratory conditions $\left(22 \pm 2^{\circ} \mathrm{C}\right.$ and $<70 \%$ relative humidity). In a randomized controlled manner, participants completed two stepwise incremental tests on separate occasions to determine anaerobic threshold (AnT) and $\mathrm{V}_{\mathrm{O} 2 \max }$ with and without LBCG. Randomized repeat 1-h time trials (1HTT) were also performed under each condition on separate days to quantify endurance cycling performance.

\section{Incremental Test}

Participants randomly completed two stepwise incremental tests to determine $\mathrm{AnT}$ and $\mathrm{V}_{\mathrm{O} 2 \max }$ in the control and LBCG conditions. Before each incremental 


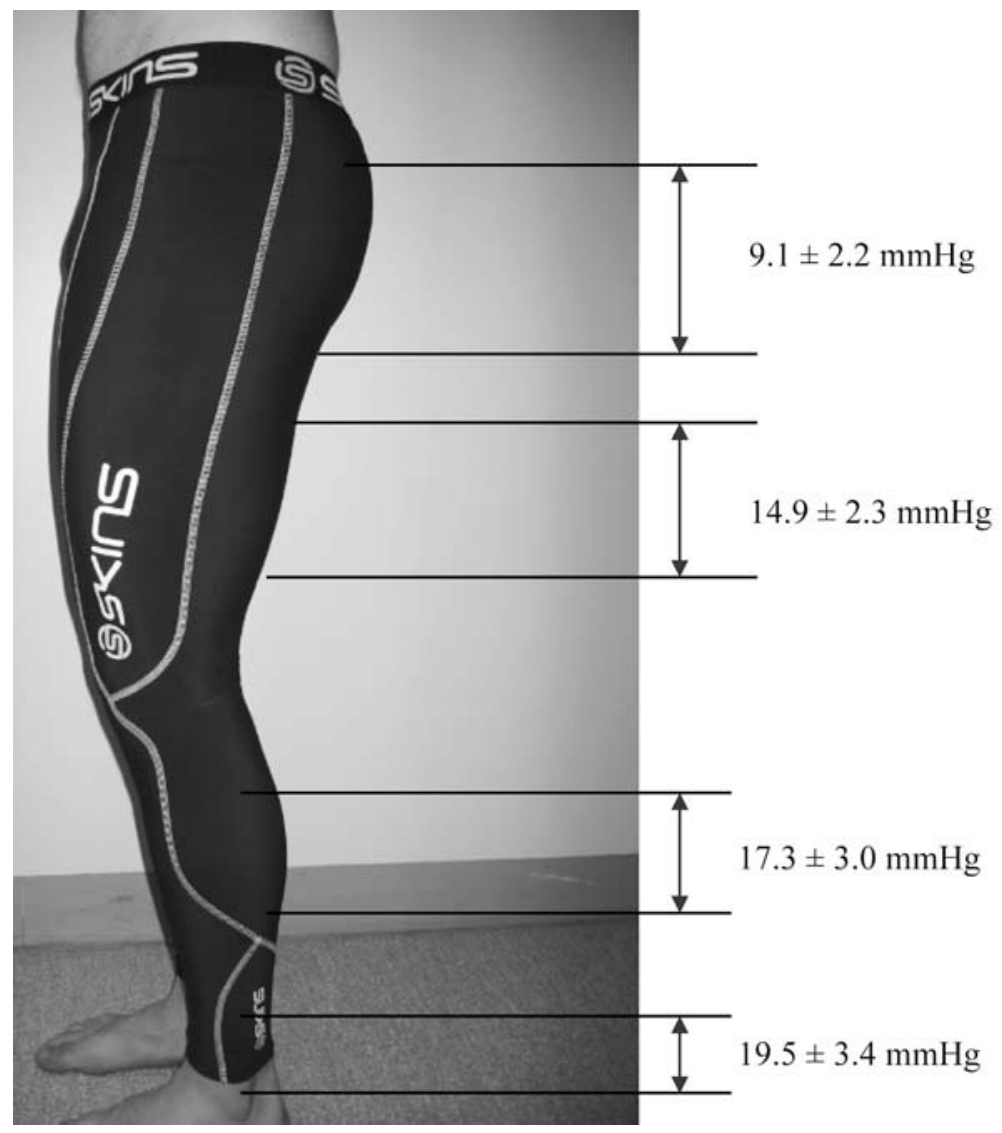

Figure 1 - The mean $( \pm \mathrm{SD})$ compression gradient of the lower-body compression garments used in the present study.

test, participants performed a warm-up of $3 \mathrm{~min}$ at $100 \mathrm{~W}$ and a further 2-min warm-up at a self-selected power output (PO). The incremental test commenced at $100 \mathrm{~W}$, and resistance was increased by $50 \mathrm{~W}$ every 3 min. ${ }^{13}$ Attainment of $\mathrm{V}_{\mathrm{O} 2 \max }$ was determined using previously established criteria, ${ }^{14}$ which involves the attainment of a plateau in $\mathrm{V}_{\mathrm{O} 2}$ (increase $<2 \mathrm{~mL} \cdot \mathrm{kg}^{-1} \cdot \mathrm{min}^{-1}$ ) despite an increase in work load, or observation of any two of the following: (1) volitional exhaustion; (2) attainment of age-predicted maximal heart rate (HR; $220-$ age; $\left.\pm 10 \mathrm{~b} \cdot \mathrm{min}^{-1}\right)$; or (3) Respiratory Exchange Ratio value $\geq 1.15$. We defined $V_{\mathrm{O} 2 \max }$ as the highest 30 -s rolling average of oxygen consumption observed at the completion of the test. We calculated AnT as the PO where the $\left[\mathrm{BLa}^{-}\right]$was equal to $4 \mathrm{mmol} \cdot \mathrm{L}^{-1}$.

\section{One-Hour Time Trial}

Participants performed randomized repeat $1 \mathrm{HTT}$ in both the control and LBCG conditions. Participants undertook a 5-min warm-up at $100 \mathrm{~W}$ and then a further 
2 min at self-selected variable PO. The PO for each subject was initially set at their condition-specific PO at AnT from the previous incremental tests. Participants were free to self-select the PO during the 1HTT using a custom-made electronic switch integrated to the ergometer control box. Participants were instructed to hold the highest PO possible while maintaining a cadence between 90 to 100 RPM throughout the 1HTT.

During the 1HTT, PO (W), cadence (RPM), and cumulative work $(\mathrm{kJ})$ were recorded at $1 \mathrm{~Hz}$ using custom-written Labview software (National Instruments, Austin, TX) on a personal computer connected to the ergometer control box. Absolute and relative measures of mean power, peak power, and total work were taken as measures of performance. The technical error of measurement (TEM) and percentage technical error of measurement (TEM\%) for mean power (TEM: $7.93 \mathrm{~W}$; TEM\%: 4.61\%) and total work (TEM: $1.492 \times 10^{4} \mathrm{~J}$; TEM\%: $0.01 \%$ ) were acceptable within this laboratory. Furthermore, cycling economy was calculated and defined as units of power output (W) per milliliter of oxygen consumed $\left(\mathrm{mL} \mathrm{O}_{2} \cdot \mathrm{kg}^{-1} \cdot \mathrm{min}^{-1}\right)$ across the 1 HTT. ${ }^{15}$ To minimize the effects of body temperature increases on circulatory responses, a mechanical fan (Coral Breeze, China) was placed directly posterior to the participants with a constant wind speed during the 1HTT for all participants.

\section{Physiological Measures}

Blood Lactate. Blood lactate concentration ([BLa $\left.{ }^{-}\right]$) was determined using capillarized blood samples drawn from hyperemic fingertips. Samples were drawn into 30- $\mu \mathrm{L}$ heparinized capillary tubes (Bacto Laboratories, Liverpool, NSW) and measured using an Accusport Lactate Analyzer (Boehringer, Mannheim, Germany). The blood samples were taken during the last $30 \mathrm{~s}$ of every work stage during the incremental tests and each 15 min during the repeat 1HTTs.

Heart Rate. Heart rate (HR) was continually recorded at 5-s intervals throughout all exercise testing using a Polar s610i HR monitor (Polar Electro, Oy, Kempele, Finland). Heart rate data were downloaded to a personal computer for posttesting analysis using Polar Precision Performance Software v4.0 (Polar Electro).

Expired Gas Analysis. Expired gas measures were continuously measured throughout the incremental tests and during the final 5 min of every 15 -min period across both 1HTT. Breath-by-breath analysis of expired gas measures were performed using a Medgraphics CPX/D system (Medgraphics, St. Paul, MN). Expired gas was collected using a preVent pneumotach (Medgraphics) and transported through sample lines, where $\mathrm{O}_{2}$ and $\mathrm{CO}_{2}$ concentrations were measured by highresponse analyzers $\left(\mathrm{O}_{2}\right.$ : Zirconia $\left[<80 \mathrm{~ms} ; \pm 0.03 \% \mathrm{O}_{2}\right] ; \mathrm{CO}_{2}$ : infrared absorption $\left[<130 \mathrm{~ms} ; \pm 0.05 \% \mathrm{CO}_{2}\right]$ ). Participants wore a mouthpiece with saliva trap and nose clip during all respiratory gas testing (Medgraphics). Before each test, the preVent pneumotach was calibrated with a 3-L syringe (Medgraphics) and the analyzers calibrated with gases of known concentrations according to the manufacturer's instructions. The reliability of expired gas measures at $\mathrm{V}_{\mathrm{O} 2 \max }$ is adequate for the testing laboratory (TEM: $0.21 \mathrm{~L} \cdot \mathrm{min}^{-1}$; TEM\%: 5.25\%). 
Near-Infrared Spectroscopy. Muscle oxygenation (mOxy) of the vastus lateralis was continuously monitored across all exercise testing using a custom-modified Runman near-infrared spectroscopy (NIRS) system. The NIRS probe consisted of six light filament bulbs, two optical filters at 760 (760fs10-12.5; Andover Corporation, Salem, NH) and $850 \mathrm{~nm}$ (850fs10-12.5; Andover Corporation), and two photodetectors (OPT301, Burr-Brown, Dallas, TX). The probe determined relative changes in oxyhemoglobin $\left(\mathrm{HbO}_{2}\right)$ /oxymyoglobin $\left(\mathrm{MbO}_{2}\right)$ and hemoglobin/myoglobin concentrations during exercise testing.

The distance diodes and photodetectors were positioned $4 \mathrm{~cm}$ apart, throughout all testing. The probe was positioned $14 \mathrm{~cm}$ from the lateral-superior border of the patella and placed over the belly of the v. lateralis. Before application of the probe, skinfold thickness was measured using Harpenden skinfold calipers (John Bull Instruments, UK) to the nearest $0.1 \mathrm{~mm}$ to ensure the signal was not distorted by excessive subcutaneous adipose tissue $(8.6 \pm 1.5 \mathrm{~mm})$. Before probe placement, the site was shaved and a clear Opsite (Smith \& Nephew, London, UK) dressing was placed on the skin underneath the photodetectors to prevent signal distortion from sweat accumulation. Once positioned, the probe was taped to the leg and securely bandaged with a dark cloth to prevent movement and ensure no visible light was detected by the photodetectors. Minimal compressive forces were applied to the thigh by the bandage to ensure the compression of the LBCG was being isolated. All leads were secured using tape to reduce any movement artifact.

The NIRS device was interfaced with a Labview NI DAQPad-6015 A/D card (National Instruments), and recorded at $1 \mathrm{~Hz}$. Custom-written Labview software (National Instruments) was used to display and record both the 760- and 850-nm signals during testing. Muscle oxygenation was calculated as the difference between the signal at the 760- and 850-nm wavelengths. Normalization of the NIRS system was performed immediately before each exercise test at both the 760- and 850-nm wavelengths. During normalization, the mOxy signal was manually adjusted to $0 \pm 0.01 \mathrm{mV}$ to ensure changes in mOxy were relative to this point. Each wavelength had variable gain and was preamplified using instrumentation amplifiers. All calibration procedures were performed with the subject in the seated position on the ergometer, with their right leg at the bottom of the crank cycle. During calibration, all NIRS signals were stabilized for at least $30 \mathrm{~s}$ before testing.

Cuff ischemia was undertaken after a 5-min recovery period to maximally deoxygenate and oxygenate the $\mathrm{v}$. lateralis to provide an ischemic-hyperemic scale. It involved the rapid inflation of a thigh cuff above the probe to $240 \mathrm{mmHg}$ to apply suprasystolic pressure. The pressure was maintained until the mOxy signal had reached a nadir for approximately 30 to $60 \mathrm{~s}$, whereupon the cuff was rapidly released to induce a hyperemic response. The nadir value of the mOxy signal was taken as $0 \%$ oxygenation, whereas the peak hyperemic response was taken as $100 \%$ oxygenation. All successful mOxy values derived from testing were normalized within this scale as a percentage. The reliability of mOxy measures at $\mathrm{V}_{\mathrm{O} 2 \max }$ is adequate for the current testing laboratory (TEM: 3.8\%; TEM\%: $10.8 \%)$. 
To calculate muscle oxygenation economy throughout the 1HTT, the mean mOxy $(\%)$ across each 15-min interval and the entire time trial was subtracted from 100 to determine the mean percentage of $\mathrm{O}_{2}$ used within the muscle. Mean power output for each time period was then divided by the corresponding percentage of $\mathrm{O}_{2}$ used $\left(\mathrm{W} \cdot \%^{-1}\right)$. The formula used was

$$
\mathrm{MOE}_{(\mathrm{t})}(\%)=\left[\mathrm{PO}_{(\mathrm{t})} /\left(100-\operatorname{mOxy}(\%)_{(\mathrm{t})}\right)\right]
$$

where $\mathrm{MOE}=$ muscle oxygenation economy, ${ }_{(\mathrm{t})}=$ specified time period, $\mathrm{PO}=$ mean power output, and mOxy = mean muscle oxygenation.

\section{Statistical Analysis}

Means and standard deviations were calculated for all descriptive physiological and performance measures. To reduce the likelihood of a Type I error, a Greenhouse-Geisser adjustment was completed to ensure the sphericity of all measures. The continuous variables were examined using a Kolmogorov-Smirnov test of homogeneity to ensure that all data were normally distributed.

Magnitude-based inferences were used to identify practically significant differences between conditions for each physiological and performance response measured during the incremental test and 1HTT. ${ }^{16}$ All magnitude-based inferences were performed using Microsoft Excel (Microsoft Corporation, Redmond, WA). The magnitude of the observed differences across conditions and time was quantified using effect size statistics $\left(\eta^{2}\right)$ as previously described, ${ }^{17}$ where 0.6 , 1.2 , and 2.0 are representative of moderate, large, and very large effects, respectively. Confidence limits for the true mean values of effects were estimated with the unequal-variances statistic computed between the control and LBCG conditions. The uncertainty in the estimate of the true mean change was presented as $95 \%$ confidence intervals. The uncertainty in the effects of CG on the physiological and performance responses was calculated with the likelihood that the true effects were practically beneficial, neutral, or harmful for the smallest worthwhile change as previously used. ${ }^{18}$ The smallest worthwhile change for the performance variables of interest in the current study was approximately equal to the typical error of measurement of the 1HTT test that was used. ${ }^{19}$ To assess the probability that the effects for the physiological variables investigated in the current study were practically significant, the smallest worthwhile changes were calculated as one-fifth of the between-subject standard deviation for each condition. ${ }^{19}$

\section{Results}

\section{Incremental Test}

The physiological and performance results from the repeat incremental tests are presented in Table 1. No practically significant differences were observed in any physiological measure or $\mathrm{V}_{\mathrm{O} 2 \max }$ between the control and LBCG conditions. However, PO at AnT was likely $\left(86: 12: 2 \% ; \eta^{2}=0.6\right)$ to be greater in the LBCG compared with the control condition. The relative power output at AnT in the LBCG condition was also likely to be greater $\left(78: 19: 3 \% ; \eta^{2}=0.6\right)$ in the LBCG condition. The mean $\left[\mathrm{BLa}^{-}\right]$curves in both conditions are presented in Figure 2. 


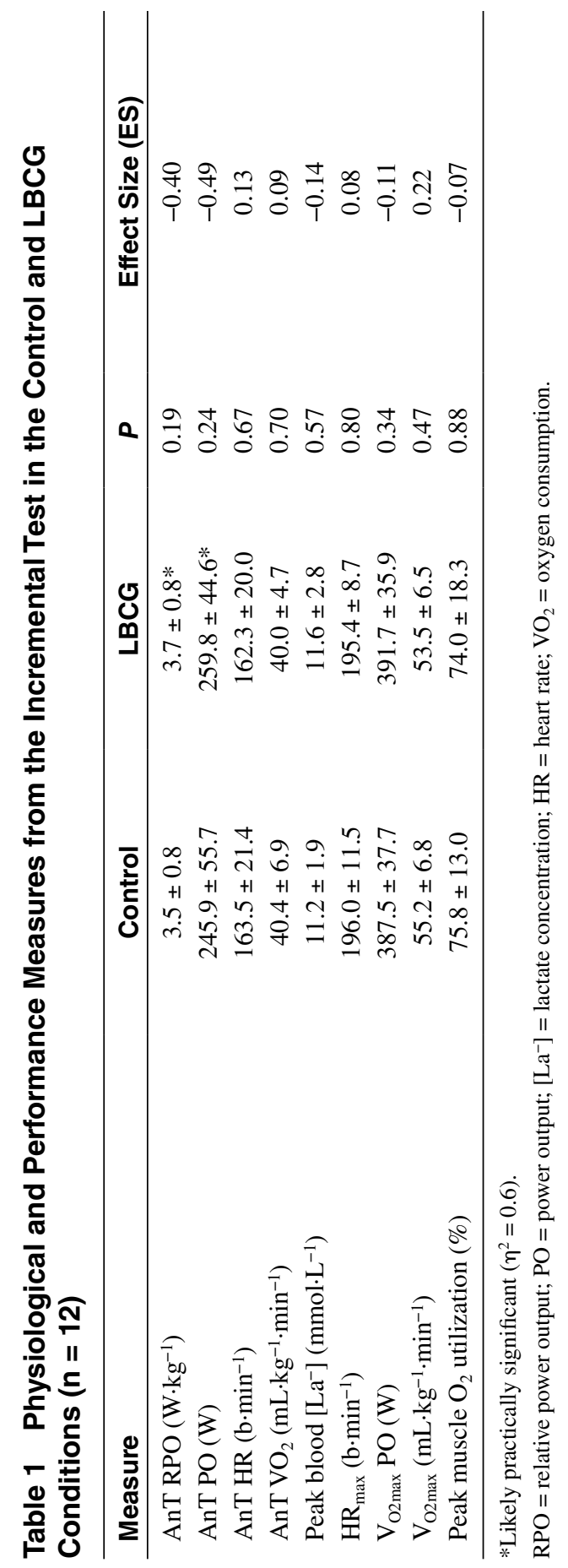




\section{One-Hour Time Trial}

The discontinuous and mean physiological and performance measures across the 1HTT are presented in Table 2. No practically significant differences were observed between conditions in any of the performance variables measured. However, the LBCG condition demonstrated likely practically greater cycling efficiency was observed within the LBCG condition across the first 15-min period of the 1 HTT when compared with the control condition $\left(81: 15: 4 \% ; \eta^{2}=0.6\right)$. Furthermore, a possibly higher mean mOxy was observed within the LBCG across the entire time trial $\left(62: 28: 10 \% ; \eta^{2}=0.6\right)$. A representative graph showing changes in mOxy data in both the control and LBCG conditions across the 1HTT is shown in Figure 3.

\section{Discussion}

The current study is the first to investigate the effects of wearing LBCG on physiological and performance responses during endurance cycling.

\section{Effects of LBCG During the Incremental Test}

Wearing LBCG produced likely practically significant improvements in both relative PO at AnT (4.2\%) and absolute PO at AnT (5.7\%). This benefit is most likely due to the proposed circulatory improvements associated with wearing LBCG. $^{3}$ The increase in PO at AnT may be related to an increased venous return and higher rate of lactate removal and metabolism in nonworking tissue that has previ-

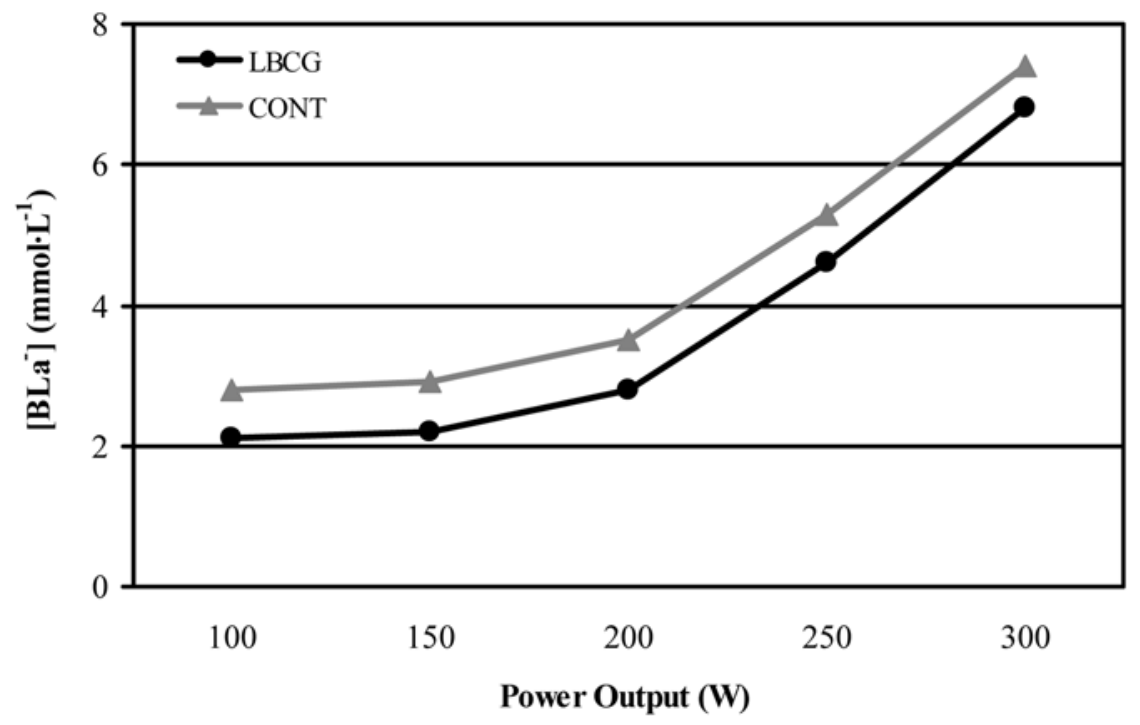

Figure 2 - The mean difference in power output at anaerobic threshold between the control and lower-body compression garment conditions. 


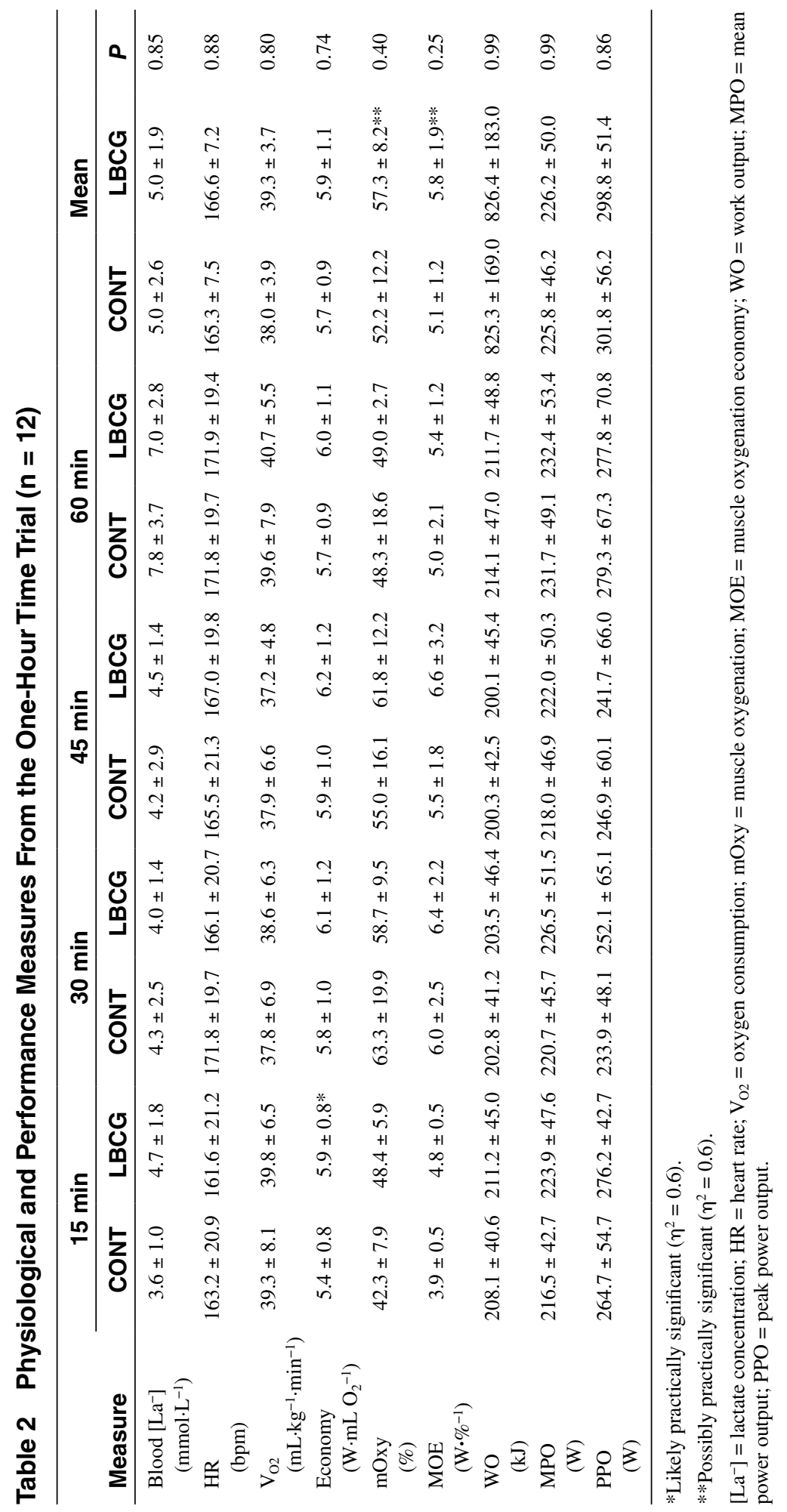




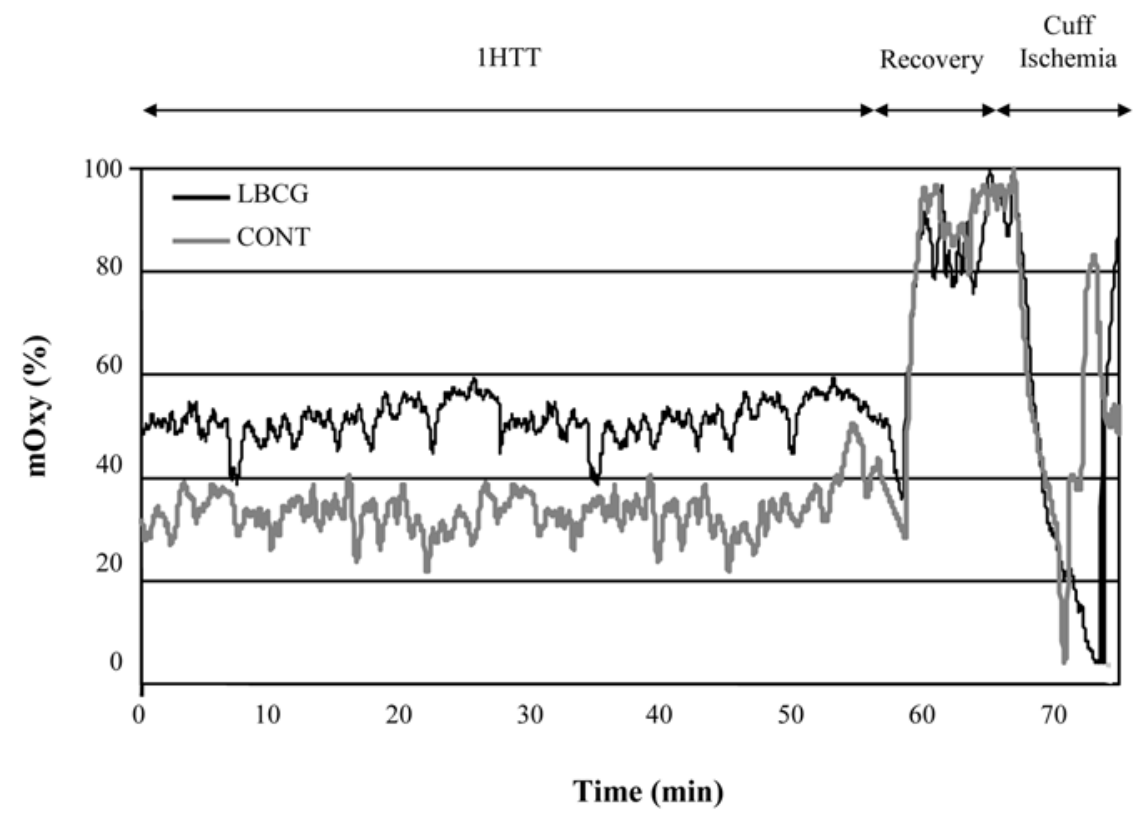

Figure 3 - Representation of the muscle oxygenation responses in the control and lowerbody compression garment conditions from a single subject.

ously been suggested to occur with wearing CG. ${ }^{3,20}$ Earlier research has reported that LBCG significantly enhance venous blood flow, suggesting an increase in the transport of deoxygenated blood and lactate toward central portions of the body.,21 Enhancements in the transport of metabolic by-products such as lactate to nonworking muscles can increase its oxidation and removal from the working muscles and circulatory system. ${ }^{3,21}$ As such, LBCG may help to sustain anaerobic metabolism during high-intensity exercise through an increased rate of lactate clearance, delaying the onset of muscular fatigue that accompanies anaerobic activity. This mechanism was supported with the observation of a lower [BLa $\left.{ }^{-}\right]$ across the incremental test with LBCG in the current study (see Figure 2). The observed rightward shift in PO at AnT with the LBCG suggests that there is potential for improved endurance performance, given the previously established strong relationship between $\mathrm{PO}$ at AnT and endurance cycling performance. ${ }^{22,23}$

Despite the improvement in AnT with LBCG, no such effect was evident on $\mathrm{V}_{\mathrm{O} 2 \max }$, which has also previously been reported to be strongly related to endurance cycling performance. ${ }^{22,24}$ The present results support previous data reporting lycra-based LBCG to have no effect on $\mathrm{V}_{\mathrm{O} 2 \max }{ }^{6}$ Bernhardt and Anderson ${ }^{6}$ suggested that the multidirectional compression exerted by the LBCG was inadequate to elicit changes in blood flow substantial enough to improve $\mathrm{V}_{\mathrm{O} 2 \max }$. However, despite the claims by these researchers that circulatory benefits should increase $\mathrm{V}_{\mathrm{O} 2 \max }$, previous researchers have observed that increases in arterial/venous blood flow do not significantly increase $\mathrm{V}_{\mathrm{O} 2 \max } \cdot{ }^{25,26}$ Furthermore, no changes in mOxy at 
AnT or $\mathrm{V}_{\mathrm{O} 2 \max }$ were observed in the current study, suggesting that LBCG have no effect on endurance athletes' aerobic capacities.

\section{Effects of LBCG on 1HTT performance}

The present data demonstrated no practically significant changes in any performance measure across the 1HTT when wearing LBCG. However, a likely practical improvement in cycling economy during the first 15-min period of the 1HTT was observed with LBCG. Similar responses with CG have been reported to occur during endurance running. ${ }^{11}$ Recently, researchers have reported that wearing LBCG increased running economy $\left(\mathrm{mL} \mathrm{O}_{2} \cdot \mathrm{kg}^{-1} \cdot \mathrm{min}^{-1} \cdot \mathrm{km}^{-1} \cdot \mathrm{h}^{-1}\right)$ during a 3-min task at a given submaximal workload of $12 \cdot \mathrm{km}^{-1} \cdot \mathrm{h}^{-1} \cdot{ }^{11}$ The researchers proposed that reductions in muscle oscillation and improvements in venous return and lactate removal were responsible for this improvement in economy. The current study observed similar acute metabolic responses during endurance cycling, which warrants the need for further investigations into the effects of CG on muscle oscillation, venous return, and lactate removal and, subsequently, economy of effort. ${ }^{11}$

Furthermore, a possible practically greater mean mOxy was sustained across the 1HTT in the LBCG condition compared with the control condition. Previous work has demonstrated LBCG to increase the utilization of $\mathrm{O}_{2}$ within working muscles during walking exercise. ${ }^{27}$ The results of the current study suggest that LBCG facilitated less $\mathrm{O}_{2}$ utilization within the working musculature, as shown by a higher level of mean mOxy across the 1HTT. Given that PO sustained during the cycling 1HTT was similar between conditions, it may be suggested that the LBCG facilitated an increase in muscle oxygenation economy as defined by a higher power $(\mathrm{W}) / \mathrm{O}_{2}$ utilization (\%) ratio (LBCG: $5.8 \mathrm{~W} \cdot \%^{-1}$; CONT: $5.1 \mathrm{~W} \cdot \%^{-1}$ ) in the LBCG condition. Previously, CG have been hypothesized to increase $\mathrm{O}_{2}$ delivery to the working muscles and subsequently facilitate greater $\mathrm{O}_{2}$ utilization. ${ }^{27}$ The difference in mOxy observed in the current study suggests that LBCG may have increased muscle oxygenation economy rather than $\mathrm{O}_{2}$ utilization within the active tissue. This observed improvement in muscle oxygenation economy offers potential benefits for endurance events that may be of longer duration and lower intensity and future research should investigate this possibility.

To date, no research has investigated the effects of LBCG on endurance cycling performance. The current study observed no improvements in performance across a 1 HTT in a group of well-trained male cyclists. Previous investigations have reported detrimental results when examining the performance effects of LBCG during endurance running. ${ }^{10,12}$ Investigators have observed LBCG to have no significant effects on $10-\mathrm{km}$ running time in 14 young male athletes. ${ }^{10}$ Furthermore, earlier research observed a significant 31-s longer 5-km run time in ten runners when wearing LBCG. ${ }^{12}$ Chatard $^{12}$ suggested that this response was related to a decreased stride length as a result of the elastic nature of the garment, limiting excessive knee extension during the swing phase of the running cycle. Due to the previously established biomechanical and muscle recruitment differences between the cycling and running modalities, ${ }^{28,29}$ this is unlikely in the current observations.

The current study identified two major factors within CG research that most likely influenced the current observations and definitely require further 
investigation. Firstly, no research has investigated the critical level of compression that is required to maximize the physiological and performance effects proposed with wearing CG. The compression of the LBCG used in the current study may have not been great enough to elicit maximal physiological and performance benefits. Secondly, no research to date has compared the differences in physiological and endurance performance responses between whole-body and lower-body compression. During cycling at intensities above $60 \% \mathrm{~V}_{\mathrm{O} 2 \max }$, the lower body has been reported to receive as much as $70 \%$ of total cardiac output and the upper body up to $30 \% .{ }^{30}$ Therefore, whole-body compression is likely to have a greater circulatory effect by acting on a greater proportion of the body, which could translate into greater improvements in endurance cycling economy.

\section{Practical Applications}

No previous research has investigated the effects of wearing CG on endurance cycling performance. The current observations suggest that LBCG offer no performance benefits during endurance cycling. However, the present data demonstrated that wearing LBCG facilitates two physiological benefits during incremental and high-intensity steady-state exercise. Firstly, the increase in PO at AnT (CONT: $245.9 \pm 55.7 \mathrm{~W}$; LBCG: $259.8 \pm 44.6 \mathrm{~W}$ ) suggests that the blood lactate production-to-clearance ratio is changed through wearing LBCG during cycling. This appears likely to be an energetic factor in terms of either efficiency or an increased oxidation of lactate within nonworking muscle. In support, improvements in cycling economy (CONT: $5.4 \pm 0.8 \mathrm{~W} \cdot \mathrm{mL} \mathrm{O}_{2}{ }^{-1} \cdot \mathrm{kg}^{-1} \cdot \mathrm{min}^{-1}$; LBCG: 5.9 $\pm 0.8 \mathrm{~W} \cdot \mathrm{mL} \mathrm{O}_{2}{ }^{-1} \cdot \mathrm{kg}^{-1} \cdot \mathrm{min}^{-1}$ ) and muscle oxygenation economy (CONT: $5.1 \pm 1.2$ $\mathrm{W} \cdot \% \mathrm{mOxy}^{-1}$; LBCG: $5.8 \pm 1.9 \mathrm{~W} \cdot \% \mathrm{mOxy}^{-1}$ ) were observed, suggesting LBCG may enable endurance cyclists to preferentially use oxidative energy sources at a performance level. These observations suggest that LBCG may delay the onset of fatigue and thus prolong optimal performance for well-trained endurance cyclists.

\section{Conclusions}

The current study demonstrated that wearing LBCG had no effects on endurance cycling performance across a 1HTT. However, LBCG were observed to elicit likely practically significant improvements in $\mathrm{PO}$ at AnT during an incremental test and possible practically significant increases in muscle oxygenation economy during a cycling 1HTT. The most plausible mechanism behind these practical benefits is improved circulation within the compressed tissue. However, future research should specifically investigate this hypothesis.

\section{Acknowledgments}

The authors would like to acknowledge the Queensland Academy of Sport Centre of Excellence for funding the project through an applied sports science research grant. Skins compression garments kindly contributed the LBCG. We would also like to acknowledge Gregory Capern for his technical assistance throughout the study, and the Rockhampton Cycling Club for the provision of participants. The results of the current study do not constitute endorsement of Skins compression garments by the authors or the journal. 


\section{References}

1. Agnelli G. Prevention of venous thromboembolism. Circulation. 2004;110:4-12.

2. Simon DA, Dix FP, McCollum CN. Management of leg ulcers. BMJ. 2004;328:13581362.

3. Chatard JC, Atlaoui D, Farjanel J, Louisy F, Rastel D, Guezennec CY. Elastic stockings, performance and leg pain recovery in 63-year-old sportsmen. Eur J Appl Physiol. 2004;93:347-352.

4. Doan BK, Kwon Y, Newton RU, et al. Evaluation of a lower-body compression garment. J Sports Sci. 2003;21:601-610.

5. Shim J, Doan BK, Popper EM, et al. The influence of lower-body compression garments on athletic performance. Med Sci Sports Exerc. 2001;33:239.

6. Bernhardt T, Anderson GS. Influence of moderate prophylactic compression on sport performance. J Strength Cond Res. 2005;19:292-297.

7. Watanuki S, Murata H. Effects of wearing compression stockings on cardiovascular responses. Ann Physiol Anthropol. 1994;13:127-137.

8. Agu O, Hamilton G, Baker D. Graduated compression stockings in the prevention of venous thromboembolism. Br J Surg. 1999;86:992-1004.

9. Stelzer JE, Moss RL. Contributions of stretch activation to length-dependent contraction in murine myocardium. J Gen Physiol. 2006;128:461-471.

10. Ali A, Caine MP, Snow BG. Graduated compression stockings: physiological and perceptual responses during and after exercise. J Sports Sci. 2007;25:413-419.

11. Bringard A, Perrey S, Belluye N. Aerobic energy cost and sensation responses during submaximal running exercise - positive effects of wearing compression tights. Int $J$ Sports Med. 2006;27:373-378.

12. Chatard JC. Elastic bandages, recovery and sport performance. Health and Protective Textiles. 1998;2:79-84.

13. Craig NP, Martin DT, Woolford S, et al. High performance track, road and mountain cyclists. In: Gore C, ed. Physiological Tests for Elite Athletes. Champaign, Ill: Human Kinetics; 2000:258-277.

14. Howley ET, Basset DR, Jr, Welch HG. Criteria for maximal oxygen uptake: review and commentary. Med Sci Sports Exerc. 1995;27:1292-1301.

15. Chavarren J, Calbet JAL. Cycling efficiency and pedalling frequency in road cyclists. Eur J Appl Physiol. 1999;80:555-563.

16. Hopkins W. Statistical vs clinical or practical significance. Sportscience; 2002:6.

17. Cohen J. A power primer. Psychol Bull. 1992;112:155-159.

18. Pyne D, Hopkins W, Batterham A, Gleeson M, Fricker P. Characterising the individual performance responses to mild illness in international swimmers. Br J Sports Med. 2005;39:752-756.

19. Spencer M, Dawson B, Bishop D, Goodman C, Dascombe B. Performance and metabolism in repeated short-sprint exercise: effect of recovery intensity. Scand $J$ Med Sci Sports., in press.

20. Berry MJ, McMurray RG. Effects of graduated compression stockings on blood lactate following an exhaustive bout of exercise. Am J Physiol. 1987;66:121-132.

21. Myers J, Ashley E. Dangerous curves: a perspective on exercise, lactate, and the anaerobic threshold. Chest. 1997;111:787-795.

22. Berran BE, Otto RM, Wygand JW, et al. Peak oxygen consumption and metabolic efficiency of cyclists. Med Sci Sports Exerc. 2006;38:241.

23. Billat VL, Sirvent P, Py G, Koralsztein J-P, Mercier J. The concept of maximal lactate steady state: a bridge between biochemistry, physiology and sport science. Sports Med. 2003;33:407-426.

24. Impellizzeri FM, Rampinini E, Sassi A, Mognon P, Marcora S. Physiological correlates to off-road cycling performance. J Sports Sci. 2005;23:41-47. 
25. Grassi B. Delayed metabolic activation of oxidative phosphorylation in skeletal muscle at exercise onset. Med Sci Sports Exerc. 2005;37:1567-1573.

26. Sirna K, Paterson DH, Kowalchuk JM, Cunningham DA. Effect of supine versus upright exercise on $\mathrm{VO}_{2}$ kinetics in young versus older adults. Med Sci Sports Exerc. 1998;30:S188.

27. Agu O, Baker D, Seifalian AM. Effect of graduated compression stockings on limb oxygenation and venous function during exercise in patients with venous insufficiency. Vascular. 2004;12:69-76.

28. Carter H, Jones AM, Barstow TJ, Burnley M, Williams CA, Doust JH. Oxygen uptake kinetics in treadmill running and cycle ergometry: a comparison. J Appl Physiol. 2000;89:899-907.

29. Bijker KE, de Groot G, Hollander AP. Differences in leg muscle activity during running and cycling in humans. Eur J Appl Physiol. 2002;87:556-561.

30. Calbet JA, Gonzalez-Alonso J, Helge JW, et al. Systemic and regional blood flow during incremental bicycle exercise to exhaustion. Med Sci Sports Exerc. 2006;38:S44. 
Copyright of International Journal of Sports Physiology \& Performance is the property of Human Kinetics Publishers. Inc. and its content may not be copied or emailed to multiple sites or posted to a listserv without the copyright holder's express written permission. However, users may print. download, or email articles for individual use. 